\title{
Los docentes de Telebachilleratos Comunitarios, perfiles que orientan la práctica y flexibilización de los procesos formativos
}

\author{
Teachers from Community Tele-Baccalaureates, \\ profiles that guide the practice and flexibility of the teaching processes \\ Marcos Jacobo Estrada Ruiz \\ Sergio Jacinto Alejo López
}

\begin{abstract}
RESUMEN
Este artículo presenta resultados de una investigación que tuvo como objetivo analizar el perfil y las condiciones de trabajo de los docentes que integran los Telebachilleratos Comunitarios (TBC) en Guanajuato, México. Particularmente nos concentramos en responder preguntas referentes al perfil de estos y su relación con el tipo de docencia asumida, así como la forma en que ese perfil y el proceso de incorporación al TBC condiciona parte de sus resultados. Se siguió el modelo CIPP (Context, Input, Process, Product) para la construcción de los instrumentos y se asumió un enfoque cualitativo desde el que se priorizaron estrategias interpretativas. Se presenta información obtenida mediante entrevistas a profundidad, realizadas a docentes de los TBC de dos municipios del estado de Guanajuato. Entre otros resultados, se caracteriza a estas instituciones como una opción educativa marcada por la precariedad. También se muestra cómo el perfil de los docentes orienta la práctica de estos y el tipo de trabajo que realizan en el aula, impactando en los procesos formativos de los estudiantes, teniendo como característica la flexibilización de tales procesos.
\end{abstract}

Palabras clave: docente, educación media, educación rural, formación docente, política educacional.

\begin{abstract}
This article presents results of a research that aimed to analyze the profile and work conditions for teachers that belong to the Community Tele-Baccalaureates (TBC) in Guanajuato, Mexico. Specially, in this paper we focused on answering questions related to teachers' profile and their relationship with the type of teaching taken on, and how their profile and incorporation process determine the results. The CIPP (Context, Input, Process, Product) model was followed for the development of the measurement tools and a qualitative approach was assumed from which interpretive strategies were prioritized. Information obtained through in-depth interviews conducted with CTB teachers from two municipalities in the state of Guanajuato are presented. Among other results, these institutions are characterized as an educational option marked by precariousness. Also, it shows how the profile of teachers guides their practice and the type of work they do in the classroom, impacting the learning processes of students and having the flexibility of such processes as a characteristic.
\end{abstract}

Keywords: teacher, secondary education, rural education, teacher training, educational policy. 


\section{INTRODUCCIÓN}

El objetivo de este artículo es analizar el perfil y condiciones de trabajo de los docentes que integran los Telebachilleratos Comunitarios (TBC) en Guanajuato, México. Interesaba saber acerca de su formación inicial, el proceso de ingreso y permanencia en el subsistema, así como la manera en que lo anterior se relaciona con el tipo de estilo docente que se asume tras su integración a dicha institución. Los TBC comenzaron a operar en el 2014, y fueron parte importante de las estrategias implementadas para ampliar la cobertura en el nivel medio superior (Guzmán, 2018), en particular en las zonas rurales del país. En México el nivel medio fue declarado como obligatorio a partir del 2012, tras una serie de reformas y cambios que modificaron de manera importante dicho nivel, y que se suman a una lógica de cambios que se dieron de forma similar en otros países del continente (Saccone, 2016).

A poco más de un lustro de su creación, importaba saber sobre las condiciones de su funcionamiento, así, lo que realizamos es, inicialmente, una caracterización del subsistema en general, pero desde uno de los actores principales, es decir los docentes. A través de estos, damos cuenta de las condiciones de creación de estas instituciones, los procesos experimentados por los profesores, la manera en que fueron contratados, su perfil, entre otros elementos, que nos hablan de procesos más profundos que se han desarrollado en los esfuerzos del Estado mexicano por incrementar la cobertura en este nivel educativo y desde los contextos rurales.

Formalmente los TBC estaban destinados a las comunidades rurales con población menor a los 2,500 habitantes, es la modalidad educativa del nivel medio que más ha crecido en el país, y entre las tres entidades con mayor crecimiento se encuentra precisamente el estado de Guanajuato (Medrano, 2019), en el cual se desarrolló este trabajo.

Varias han sido las preguntas que se abordan en el estudio amplio, para este artículo el foco está en responder: ¿Cuál es el perfil de los docentes y cómo se relaciona con el tipo de docencia asumida? ¿De qué forma el perfil y el proceso de incorporación de docentes al TBC condicionan parte de sus resultados?

Marcos Jacobo Estrada Ruiz. Profesor-investigador del Departamento de Educación de la Universidad de Guanajuato, México. Es doctor en Educación por la Universidad Autónoma del Estado de Morelos (UAEM), licenciado en Docencia en Ciencias Sociales y Humanidades por la misma universidad y tiene una especialidad en Migración Internacional por el Colegio de la Frontera Norte (COLEF). Cuenta con Perfil Prodep y es miembro del Sistema Nacional de Investigadores, Nivel II. Correo electrónico: marcos.estrada@ugto.mx. ID: https://orcid.org/0000-0002-4947-208X.

Sergio Jacinto Alejo López. Profesor de tiempo completo en el Departamento de Ingeniería Agroindustrial de la Universidad de Guanajuato, México. Es doctor en Ciencias de la Educación por la Universidad Autónoma del Estado de Hidalgo (UAEH) y maestro en Investigación Educativa por la Universidad de Guanajuato. Perfil ProdeP y candidato a Investigador Nacional en el Sistema Nacional de Investigadores. Correo electrónico: jacintosergio@hotmail.com. ID: https://orcid.org/0000-0001-8217-7050. 


\section{LOS DOCENTES Y LA “NUEVA" RURALIDAD}

El subsistema de TBC se incorpora a comunidades rurales dispersas y con poca población, que en general adolecen de servicios públicos en suficiencia y calidad, y presentan diversas desventajas en sus condiciones sociales, económicas, políticas y culturales, bajo el denominador común de la pobreza. La vocación de las comunidades rurales de esta naturaleza es heterogénea, por ejemplo, en el entorno de Guanajuato, en la actividad agrí́cola solo se va a encontrar el cultivo de cereales y granos básicos de temporal, ganado de pastoreo en terrenos de agostadero principalmente, acentuados flujos migratorios especialmente hacia los Estados Unidos de parte de adultos y jóvenes varones. También en el contexto de este estudio prevalece la composición de familias con mujeres mayores, dedicadas al cuidado del hogar o en trabajos de maquiladoras cercanas, viviendo igualmente a expensas de las remesas que reciben. Desde hace algún tiempo, estas comunidades cada vez más están vinculadas a la vida urbana, interactuando y replicando comportamientos sociales que en el pasado no se daban. Tal es el caso del trabajo en actividades secundarias y terciarias, en el interés de las empresas por aumentar su participación en los mercados y el aseguramiento del recurso humano barato, el uso de tecnologías de la comunicación, el abandono de parte de estas poblaciones de las actividades económicas primarias. Así, la nueva forma de vida en el campo, como dice De Grammont, "incorpora ahora una diversidad de actividades y relaciones sociales que vinculan estrechamente las aldeas campesinas con los centros urbanos y la actividad industrial" (2004, p. 279).

Estas actividades propias de la globalidad y del poder económico multinacional, conocidas como una nueva ruralidad y desarrolladas más allá de la autoridad de los Estados-nación, contrarrestan la hechura de políticas públicas democráticas de legitimación social. Aparecen nuevas reglas del juego dando lugar a territorios con un amplio abanico de aspectos a considerar, como son los bienes simbólicos, lenguas, arte, comidas, producciones no-agrarias, servicios, etc. (Giarracca, 2001).

En esta dirección de la nueva ruralidad, se ha dado la extensión de la educación básica obligatoria y gratuita, particularmente con el establecimiento de los TBC para la formación ciudadana de la población joven de estas localidades, y buscando, a través de estos, garantizar un mínimo de igualdad de oportunidades. Sin embargo, son evidentes las diferencias en la enseñanza y el aprendizaje del estudiantado, distancias que están conformadas por las desigualdades socioculturales de las condiciones familiares (Bourdieu, 1987).

Los TBC en las localidades rurales parecen desconocer el desarrollo de las formas de la cultura local, proporcionando saberes disciplinares, fragmentados y homogéneos, venidos de sus currículos formales, provocando contradicciones y tensiones con la realidad vivida de los actores (Weiss, 2017), así también como el desinterés y desapego de las y los jóvenes por el estudio, prefiriendo otras actividades, como el 
trabajo, apoyando en el hogar, en la sociabilidad comunitaria con los pares jóvenes, o bien estableciendo relaciones de pareja más allá de la familia.

Aunada a la ampliación de la cobertura en la educación media superior (EMS) mediante la instauración de los TBC, estos sobrellevan en gran medida lo que sucede en la educación rural del nivel medio en nuestro país. En el resumen del foro "Propuestas para la educación rural durante el sexenio 2018-2024" (RIER, 2018) se menciona que en las comunidades rurales persisten las dificultades en la asignación y aplicación de recursos para garantizar la calidad educativa y el derecho a la educación; los procesos de enseñanza se han subordinado a modelos urbanos, en este sentido, el papel de los docentes se centra en labores directivas y administrativas, al mismo tiempo que imparten sus clases, y con poco arraigo en la vida de la comunidad; mientras prevalece una importante necesidad de fomentar más el aprendizaje colaborativo, con un enfoque comunitario, articulado con el trabajo, así como el diseño de estrategias didácticas situadas en un currículo más flexible (RIER, 2018).

En los TBC sucede lo que menciona Pérez (2004), en el sentido de encontrarse la escuela en un escenario de incertidumbre y ambigüedad. También en lo referente a las finalidades sustantivas que delimitan su tarea educativa a un espacio en el que ya no se toman las decisiones trascendentes, ni es la unidad de cambio, perdiendo su espacio singular de interacción social. Tampoco parece ser el profesor el que decide sobre la didáctica empleada, ya que es solamente un profesional (experto provisional y emergente) al servicio de la institución, con una libertad limitada, sujetándose al proyecto curricular y al ideario ideológico de la escuela, dejando de lado la cultura como docente.

Los docentes no solamente se encuentran frente a las imposiciones del sistema educativo para cumplir con las políticas, valores y condiciones establecidas a través de la institución escolar, también se enfrentan con el estudiantado, pues estos comprenden la escuela muy diferente: la consideran, en muchas de las ocasiones, no como un lugar de conocimiento y aprendizaje, sino como un espacio de socialización, donde despliegan su condición juvenil que entra, las más de las veces, en contradicción con la institución y los profesores. Sin embargo, no deja de concebirse al docente como "el factor inmediato de mayor importancia en la determinación de la calidad de los procesos educativos" (Pérez, 2004, p. 164).

Estas imposiciones del sistema educativo y escolar a las que se enfrenta el docente tienen su explicación, según Pérez (2004a), en el escenario de la cultura docente, conformada por distintos niveles de valores, como son un nivel representado por las creencias y códigos éticos, otro nivel más racional en el que se destacan las normas y expectativas del contexto social, y un tercer nivel en el que sobresalen los sentimientos y preferencias personales; dicha cultura docente forma parte importante de la cultura de la escuela. De esta manera, el docente se encuentra viviendo tensiones ante las exigencias de un tejido social incierto, un despliegue acelerado de las tecnologías, 
además de una subordinación del libre mercado, y, sobre todo, agrega el autor, de un sistema escolar inflexible, opaco y burocrático, en el cual "los docentes se encuentran cada día más inseguros e indefensos, se sienten amenazados por una evolución a la que no pueden responder o no saben responder" (Pérez, 2004a, p. 163).

En la situación que vive esta cultura docente en los TBC, resulta importante no dejar de lado las características de la nueva ruralidad, como es la incorporación de los jóvenes a pautas familiares y laborales como la emigración hacia los centros urbanos y hacia los Estado Unidos de Norteamérica, apartándose de sus preferencias de vida, la vocación escolar, poniendo en entredicho el papel de la escuela en cuanto a lo que señala Pérez (2005), de la encomienda social de la preparación de los individuos de las nuevas generaciones, para que en un futuro logren incorporarse al mundo del trabajo. También la función de socialización de la escuela, para la construcción de una ciudadanía en los individuos que les permita su participación en la vida pública y la composición de las instituciones en el tejido social. Menciona el autor que, en dicha socialización, la economía y sus leyes de oferta y de demanda crean claras y escandalosas diferencias entre los individuos y los grupos, que plantean requerimientos que contradicen los procesos de socialización en las escuelas (Pérez, 2005, p. 20).

Estas diferencias y desigualdades impactan en la forma de efectividad de los TBC, así como en la cultura docente, particularmente en el cumplimiento de sus distintas funciones relativas a su preparación, capacitación, en el desempeño de actividades de administración, y con el medio social de la escuela. Además, en dicha cultura, los docentes padecen tanto la limitación de recursos económicos y pedagógicos como también de necesitar un mayor apego personal y profesional al sistema escolar del TBC, a las tradiciones de la comunidad local y de adaptación para desarrollar un trabajo colaborativo con sus pares. Todo este bagaje de carencias en el docente se despliega sin la garantía de una estabilidad laboral, ni la incorporación segura a una trayectoria dentro del sistema educativo.

\section{LOS ANTECEDENTES INVESTIGATIVOS}

El TBC como opción educativa, en el marco de la educación media en México, encuentra sus antecedentes históricos en los Telebachilleratos que, en algunas entidades como Veracruz, se desarrollaron en los años 80 del siglo pasado (Salazar, 2007; Espinoza, 2014). Aunque hay importantes diferencias, tienen una novedad que marca un cambio significativo respecto a los anteriores, es decir, la adjetivación en el currículum de lo comunitario. Fuera de eso, su estructura parece ser similar: funciona en las instalaciones de otros niveles, en especial de las telesecundarias; tiene una estructura docente mínima, tres profesores, de los cuales uno está contratado con 30 horas y realiza funciones de director encargado, mientras los otros dos tienen una contratación de 20 horas, dedicados a la docencia. Entre sus características 
principales se encuentra el hecho de que es una opción educativa que estaba pensada para la atención de comunidades menores a 2,500 habitantes. Sin embargo, aunque lo anterior no se ha cumplido del todo, sí se ha dedicado a atender a población de los contextos rurales (Estrada y Alejo, 2018). En términos generales puede caracterizarse al TBC como una opción educativa del nivel medio superior, que tiene entre sus objetivos principales el incremento de la cobertura en las zonas más marginadas del país. Además, fue concebida desde las propias políticas del subsistema, como una respuesta de equidad para estas poblaciones (SEMS, 2016).

Algunos trabajos de investigación han empezado a mostrar resultados sobre los TBC, por su corta historia no abundan investigaciones que den cuenta de su impacto y funcionamiento, así como de las perspectivas de los actores. Aunque también es cierto que, a poco más de un lustro de su creación, hay un leve incremento de estudios, aunque no en lo que en este texto se reporta, es decir, en la perspectiva de los docentes.

Lo que existe actualmente es referente a su caracterización; destacan los trabajos de Estrada y Alejo (2018; 2019), en los que caracterizan a los TBC de una entidad del país, desde sus condiciones iniciales de apertura y el desarrollo que han tenido. También hay trabajos que dan cuenta del impacto en las perspectivas de los jóvenes y de la comunidad, a partir de la instalación de esta opción educativa en los contextos rurales (Estrada y Razo, 2019).

Entre los estudios existentes destacan los informes institucionales, particularmente a partir de lo que era el Instituto Nacional para la Evaluación de la Educación (INEE), como ejemplo los trabajos realizados por Weiss (2017; 2017a), en los que muestra, entre otras cosas, cómo se instalaron los TBC en distintas entidades del país, la presencia de los mismos en contextos urbanos, indicándonos, por una parte, la prevalencia de alta demanda de estudios en la EMS y, por otra, que estas instituciones, al entrar en funcionamiento de manera rápida, empezaron a ser demandadas también por los contextos para los cuales originalmente no estaban destinados, como la población urbana. También se ubicaron las condiciones adversas que han encontrado para su funcionamiento, como la desconfianza de las comunidades acerca de su función y permanencia. Se ha caracterizado a este modelo como un subsistema joven con docentes jóvenes, es decir, está integrado, en general, por jóvenes recién egresados de diferentes carreras y que cuentan con poca experiencia profesional (Weiss, 2017a). En el mismo sentido, el trabajo de Guzmán (2018) refleja que los docentes de este subsistema son jóvenes, comparados con el resto de las opciones en la educación media. Del mismo modo, en el TBC los docentes tienen la escolaridad más baja, también son los que más alto porcentaje tienen de falta de correspondencia entre su perfil profesional y las actividades docentes y disciplinares que realizan en estas instituciones (Estrada y Alejo, 2018). 
Lo anterior naturalmente parece traer consecuencias, así, el trabajo de Leyva y Guerra (2019) destaca cómo los docentes, al presentar un perfil y formación alejada al tipo de trabajo que desarrollan, los lleve a que inviertan más tiempo en la preparación de sus clases; aunque también es cierto que lo anterior repercute en que se presente un cierto trabajo colaborativo, al tener que recurrir a la ayuda de los pares. Por otra parte, las autoras encuentran que estos docentes siguen, como estrategia institucional, dedicar la primera semana de clases a subsanar conocimientos y habilidades que los jóvenes no tienen en este nivel y que les son necesarios para un mejor desempeño e integración. Además destacan la importancia que tienen las estrategias de relación con el entorno comunitario, mismas que se revelan como una característica fundamental de los TBC.

Para finalizar este apartado hay dos trabajos importantes, uno el de Cortés, Peniche y Crescencio (2019), quienes hacen un análisis de las prácticas de liderazgo escolar, encontrando entre las más habituales las de la motivación al personal docente sobre el proyecto institucional, la gestión pedagógica, el rediseño de la organización y la promoción del desarrollo profesional, entre otras, todas estas acciones volcadas hacia la motivación de los docentes para lograr un trabajo colaborativo. El otro trabajo es el de Landín y Arriaga (2019), que es básicamente una propuesta de atención a la formación pedagógica de los docentes del TBC en Guanajuato. Con base en la realización de una evaluación diagnóstica, centrada en los conocimientos y habilidades necesarias para la práctica docente, obtiene como resultados que cada responsable de plantel y los docentes integraran sus planeaciones didácticas de clase. Entre otras conclusiones, los autores mencionan que, a partir de las estrategias desarrolladas, se consiguió reforzar en los profesores las prácticas y apuntalar las competencias en los procesos de planeación y en la relación enseñanza-aprendizaje.

\section{Metodología}

\section{Enfoque}

En el estudio amplio del cual se desprende este artículo, se siguió el modelo CIPP (Context, Input, Process, Product) de Stufflebeam y Shinkfield (1987), que sirvió además de guía para la construcción de los instrumentos, también para la organización de los datos. Asumimos un enfoque cualitativo (Strauss y Corbin, 2002), con la intención de arribar a hallazgos sin centrarnos en una lógica de cuantificación y priorizando, más bien, estrategias interpretativas. Se aplicó un cuestionario al estudiantado y se realizó una selección basada en criterios (Goetz y LeCompte, 1988) para los casos en los que se trabajó; por los objetivos perseguidos en este texto, aquí se presenta únicamente la información que se desprende de las entrevistas a profundidad, realizadas a profesoras y profesores de los Telebachilleratos Comunitarios de dos municipios del estado de Guanajuato (Juventino Rosas y Abasolo) durante el primer 
semestre del 2018. Si bien la muestra y el trabajo de campo realizado es más amplio (13 municipios), consideramos que lo aquí reportado es una muestra representativa de lo que finalmente se terminó encontrando para el caso de los docentes.

\section{Técnicas de recolección}

Las entrevistas resultaron útiles para acceder y comprender la perspectiva de los actores consultados, además, como sostienen Álvarez y Jurgenson (2003), esta herramienta es básica para analizar los significados de las experiencias acumuladas. Las entrevistas a profundidad con los docentes ofrecieron, comparativamente, el acceso a los significados profundos de estos actores, más que una mirada panorámica, como podría serlo una encuesta rápida (Angrosino, 2012). En promedio duraron poco más de una hora, en su mayoría se realizaron de manera individual, aunque en algunas ocasiones los directores responsables de los centros optaron porque realizáramos entrevistas grupales, es decir, a los tres docentes al mismo tiempo, conformando así, por momentos, una combinación y cruce de perspectivas que resultó enriquecedora para la recopilación de la información.

Las entrevistas realizadas a profesoras y profesores proporcionan datos que contribuyen a la caracterización de su perfil y de la lógica y dinámica de trabajo en los TBC. También posibilitan acceder a las perspectivas que los actores tienen sobre el mismo subsistema. Las dimensiones que se exploraron en las entrevistas, a través del guion general construido, versaron sobre: a) lo concerniente a las condiciones de contratación, b) los procesos de formación y actualización docente, y c) la forma de trabajo pedagógico y las estrategias en aula.

En los dos municipios considerados se eligieron dos escuelas, en las que se entrevistó a los docentes que integraban a las mismas. Como se mencionó, los TBC tienen una estructura docente mínima, conformada por tres docentes, los cuales fueron entrevistados, accediendo así a 12 actores de la comunidad escolar consultados.

\section{Procesamiento de análisis}

El trabajo de análisis para la parte de los datos de campo que se presentan pasó por los procesos habituales de sistematización y reducción, es decir, transcribimos las entrevistas en un procesador de texto y las organizamos por institución y actor. Posteriormente se realizó la búsqueda de patrones emergentes (Bertely, 2000) que permitieron la ubicación y construcción de las categorías bajo las cuales se exponen los datos, y que terminaron constituyendo parte de los hallazgos. Así, las categorías encontradas bajo este esquema y que exponemos de manera sintética fueron: a) acceso y contratación docente, b) el perfil que orienta la práctica, c) capacitación y actualización, d) entre la docencia y la administración, y e) trabajo docente y flexibilización. 


\section{Resultados}

\section{Condiciones de acceso y contratación docente}

La historia de la gestación del TBC puede rastrearse desde el análisis de varias dinámicas, una de las más emblemáticas es la de la incorporación de la plantilla docente, y lo es porque este proceso parece que ha condicionado parte de los sentidos y logros del TBC en general. Es decir, en el tratamiento que se dio a las formas de contratación y desarrollo del profesorado se puede encontrar la raíz de los resultados actuales, pero, como se verá, hay varios matices necesarios.

El proceso de contratación puede inferirse si se considera a las políticas y normas bajo las que se fundó el Telebachillerato Comunitario en el país (SEMS, 2016), y desde el ámbito de lo local en la manera en que ha ido evolucionando en la entidad a la que pertenecen los casos analizados. Es decir, por una parte, dinámicas de contratación que no están del todo destinadas a captar las habilidades docentes, pues en la mayoría de los casos no las traen, y por la otra, una contratación cada vez más en manos de la entidad encargada, implementando sus propios procesos. Es importante destacar que esto es una muestra de cómo, en el camino, estas instituciones han venido aprendiendo y desarrollándose (véase tabla 1).

El proceso de contratación bajo el cual se convoca a los docentes ha ido cambiando desde la implementación de los TBC, y varía en cada municipio, ya que las exigencias se adaptan a las condiciones tanto de los docentes como del plantel. Resulta relevante comentar que no es propiamente un concurso de oposición, como funcionan algunas de las otras instituciones de educación media en el país, lo cual marca una diferencia importante. Por ejemplo, se menciona que una de las características del proceso se basa en la cercanía entre el lugar de residencia del profesorado y la ubicación del Telebachillerato; no obstante, y previo a esta selección, los interesados han de acudir a las oficinas centrales para entregar documentación que avale su formación y los presente como aptos para el puesto. Posteriormente se les aplican exámenes

Tabla 1. Dos procesos de contratación.

\begin{tabular}{ll}
\hline \multicolumn{1}{c}{ Las primeras contrataciones } & El proceso actual \\
\hline "Fui a [la ciudad de] León por medio de otra empresa que se & "Acudimos al departamento de recursos humanos de la \\
dedicaba a contratar a las personas y fue la forma en que yo entré". & universidad [UVEG"] que puede ser en las oficinas [...], \\
"en ese tiempo hubo una empresa que se contrató, ahí fue donde & $\begin{array}{l}\text { entrega uno la documentación con currículum vitae, con } \\
\text { toda la documentación comprobatoria de trabajo, de }\end{array}$ \\
se hizo todo el procedimiento, allá me hicieron lo que son los & $\begin{array}{l}\text { experiencia, cursos, títulos, cédulas (EDATBC6)". } \\
\text { institución (EDATBC10*)". }\end{array}$
\end{tabular}

* La Universidad Virtual del Estado de Guanajuato (UVEG) es la encargada de atender, en la entidad, a los TBC.

** Claves asignadas a los datos de campo. 
relacionados al área de conocimiento a la que se enfocan; aunque, como se puede ver en la tabla 1, al inicio fue distinto y delegado en instancias externas, lo que muestra la manera en que, desde los sistemas locales, se tuvieron que generar capacidades en un corto tiempo para atender a estas instituciones, sin contar con una guía genérica nacional. Es decir, en su origen había ya una suerte de descentralización, abonando a la ya de por sí existente dispersión y heterogeneidad del sistema de educación media (Macías y Valdés, 2014).

Además del proceso formal de contratación, entraron en juego otros elementos de carácter más informal que tenían que ver con la ubicación de las escuelas. Así, cuando hubo posibilidad, los profesores solicitaban ser asignados a escuelas cercanas a sus lugares de residencia, y cuando lo anterior no se podía, lo intentaban en los semestres siguientes. A ello obedece que, en las visitas de campo realizadas, en ocasiones la planta docente estaba incompleta, pues se había dado un cambio al respecto. Este no es un elemento sin importancia, todo lo contrario, pues está en la base de lo que es el Telebachillerato Comunitario actualmente, y en este caso se muestra la parte del esfuerzo adicional y claramente de inequidad que el subsistema tiene con los docentes, pero que ha afectado todo el proceso educativo y que se consagra en los resultados de aprendizaje de las y los estudiantes, en los que, consistentemente, estas instituciones presentan los menores logros educativos (Backhoff, Guevara, Hernández y Sánchez, 2018).

\section{El perfil que orienta la práctica: perfiles variados, orígenes diversos}

Aun cuando en este artículo se toman como casos las escuelas de dos municipios, los datos en este punto coinciden con el resto de los casos considerados en el estudio amplio, es decir, los perfiles variados de los profesores que integran a los TBC, y también en algunos casos las actividades o trabajos que realizaban antes de dedicarse a la docencia (véase tabla 2).

Tabla 2. Formación inicial de los docentes del TBC.

\begin{tabular}{ll}
\hline Formación inicial & Trabajo antes del TBC \\
\hline Ingeniero en Sistemas Computacionales & En una constructora \\
\hline Licenciado en Comunicación & En la industria \\
\hline Licenciado en Administración & En una empresa \\
\hline Ingeniería Metalúrgica & Trabajos meramente administrativos \\
\hline Maestría Enseñanza de Nivel Medio Superior & Maestro de inglés en escuela privada \\
\hline
\end{tabular}

Licenciatura en Matemáticas

Licenciada en Derecho

Aunque son más los profesores consultados en este estudio, las carreras se repiten por lo que solo se ponen una vez como representativas de los perfiles profesionales de los docentes.

Fuente: Construcción personal. 
Dentro de los Telebachilleratos Comunitarios la mayoría de los profesores no cuenta con una formación totalmente enfocada a la docencia, su formación inicial se centra en áreas de conocimiento que guardan, parcialmente, relación con los campos disciplinares que ofrece el TBC, y en los cuales parecen haber sido asignados para impartir sus clases. Sin embargo, en algunos casos, también es de destacarse que, al no tener el perfil docente requerido, se han visto en la necesidad de cursar posgrados que los habiliten para dichas tareas, relacionados con la educación o con el nivel educativo en el que se encuentran.

Lo anterior tiene implicaciones importantes, mismas que destacan en este proceso, nos referimos al peso que tiene la formación inicial. Es decir, dependiendo de lo que se haya estudiado, es el enfoque o el tipo de conocimiento que orienta la práctica pedagógica del profesorado, por ejemplo, este maestro con experiencia en la industria lo comenta de la siguiente forma:

Les hablo [a los estudiantes] de salud, el tomar refresco contiene químicos, contiene el dióxido de carbono y les puede provocar diabetes, entonces ellos van viendo que no solo tenemos conocimiento en la escuela sino también en lo fáctico de las empresas, donde he estado por años [EDATBC].

Además de lo anterior, recurrir a su experiencia y disciplina de formación inicial y formarse en posgrados con sus propios recursos les ha implicado en algunos casos realizar un ejercicio de autoformación y actualización permanente. Así, comentan estar constantemente leyendo sobre diversos temas relacionados con las materias o áreas de las que son responsables, por ejemplo, de historia universal, de México, de temas económicos, de sicología, entre otros. Lo anterior se puede entender no solo como parte del proceso cotidiano del ejercicio docente, sino también, como se puede apreciar en la experiencia laboral previa, en la mayoría de los casos no se contaba con experiencia docente, y tienen entonces que dedicarle más tiempo a formarse y preparar sus clases, es decir, a generar una cierta cultura docentes mediada por distintos niveles de valores, entre otros, el de responder ante su actual profesión (Pérez, 2004a). Así, el ingreso al TBC representó para la amplia mayoría su primera incursión en dicha labor, y ante ese hecho se han visto impelidos a formarse en los contenidos que imparten.

\section{La capacitación y actualización docente}

Los procesos relacionados con los docentes, empezando por la contratación y luego su asignación a alguna escuela, tuvieron como característica inicial la delegación a otras instancias externas a los Telebachilleratos Comunitarios, o bien se tuvieron que generar capacidades de atención. Así, en general, los docentes mencionaron recibir cursos de capacitación desde su entrada a este subsistema, al menos bajo dos vertientes. 
Por un lado, lo que implica conocer y trabajar en esta modalidad y todos los procesos y áreas que lo integran. Entre otros, comentan que los cursos recibidos son sobre:

Los horarios que vamos a tener, de cómo se rigen los departamentos de recursos humanos, cómo funciona el sistema para capturar calificación, en mi caso que soy el responsable [de dirección] pues sí me dan un poquito más de cursos en cuanto a la administración del Telebachillerato [EDATBC2].

Por otro lado está lo que se puede decir que impacta en la formación de los docentes y los habilita para su trabajo en aula, o del proceso enseñanza-aprendizaje, el más importante por las implicaciones que tiene para la mejora de los aprendizajes. En este sentido, los docentes comentan estar recibiendo constantemente cursos de este tipo; así, por ejemplo, mencionan

...cursos para el manejo de la tablet que se entrega siempre en nivel medio superior, cursos para planeaciones argumentadas, cursos de formación docente" [EDRC3].

Del mismo modo, parece que los cursos que también se promueven están dirigidos a áreas estratégicas que van más allá de la docencia, y resultan importantes para la institución, pero también sobre la cual hay mayor necesidad de formación para saber cómo intervenir, tal es el caso de quienes se encargan de las tutorías:

He tomado bastantes cursos de prevención de adicciones, tomé el de herramientas para el docente, para las planeaciones, cuando entré pues sí entré así como en blanco [EDRC8].

Lo que revelan los discursos del profesorado, además de lo señalado, es lo que ya se puede inferir desde los primeros datos acerca de la formación profesional de los docentes: no tienen formación cercana a la docencia, y han tenido que solventar, parcialmente, dicha ausencia de formación con los cursos que desde los responsables de los TBC se les imparten. Pero ciertamente, en tanto procesos formativos, pueden resultar insuficientes para lo que se requiere de un profesional de la educación que, adicionalmente, se encuentra inserto en los contextos más adversos.

Ahora bien, para una de las áreas más importantes expresada en una asignatura en concreto, que es lo que se ha denominado como su signo de distinción (Bourdieu, 1988; Estrada y Alejo, 2018), la materia de Desarrollo comunitario, la respuesta parece ser la misma:

Yo soy del área de comunicación, y a nosotros nos ponen cursos aparte porque tenemos la materia de Desarrollo comunitario, donde hacemos un proyecto aquí en TBC, lo que he tomado han sido sobre inglés y sobres las tecnologías, tabletas, es lo que he tenido yo [EDRC1].

Aunque la respuesta se inscribe bajo la misma lógica de lo expresado por docentes de otras áreas, lo notable es que no se percibe una formación específica para una materia como Desarrollo comunitario, que es definitoria en estas instituciones. Así, lo que resalta es que los docentes aprovechan los cursos disponibles o los que 
les interesan por distintas razones, sin que se relacionen con lo que hacen cotidianamente o con lo que represente una problemática educativa o de necesidad formativa; por ejemplo, los cursos sobre las nuevas tecnologías, ausentes en general en los TBC pues en la mayor parte de las comunidades no hay internet, y en algunas tampoco hay luz eléctrica. Sin embargo, no deja de llamar la atención que sean cursos que les interesen y que los tomen.

En otro sentido, existe la detección de necesidades a ser atendidas; desde el trabajo de tutoría consideran, por ejemplo, que deben abordar las problemáticas que de distinto modo ubican, como los embarazos y el consumo de drogas. Sin embargo, el tipo de abordaje nuevamente revela la idea de formación que se tiene y en parte la experiencia docente con la que cuentan, principalmente en el tipo de estrategias que implementan para la atención a estas problemáticas:

...hay muchos embarazos, pues hay que meter algo de embarazos, o que hay mucha drogadicción, o sea, como meter pláticas, conferencias, lo que sea para atacar ese problema, y se puede manejar grupal o incluso individual la tutoría [EDRC?].

\section{Entre la docencia y la administración}

Hay una relación importante entre las actividades que el profesorado realiza más allá de la docencia y que entran en juego con lo que es su actividad principal, es decir, todo lo que tiene que ver con la parte administrativa y su posible impacto en lo que se considera que es su función primaria, la docencia. Por ejemplo, en el caso de los directores encargados, la labor y los tiempos dedicados es a la función administrativa fundamentalmente. Por lo narrado por los actores, no se ve cómo puedan dedicarse a la parte de la docencia, estaríamos hablando de que hay, en realidad, dos docentes (de tres) que pueden dedicarles más tiempo a esas funciones. Este aspecto también ha sido señalado en los estudios realizados por Weiss (2017).

Lo anterior pese a que sostengan que gran parte de lo administrativo es virtual. Sin embargo, diversos procesos tienen que pasar por un trabajo previo de los docentes que, como vemos, los lleva a dedicarle tiempos importantes de sus actividades:

Toda la administración del Telebachillerato se hace de forma virtual, me refiero a que ellos [los estudiantes] me traen su acta de nacimiento, comprobante de domicilio, y esa documentación yo tengo que digitalizarla y subirla al sistema para cada alumno y allá se encargan de validarla, en caso de que haya una equivocación, todo es a través de correo, por medio del teléfono [EDRC11].

Los docentes encargados de plantel tienen una contratación de tres cuartos de tiempo, es decir 30 horas semanales para la docencia y las actividades administrativas de la dirección. Sin embargo, podemos observar que, en la misma descripción de las actividades que hacen, el tiempo dedicado a lo administrativo necesita más de lo que tienen contratado: 
Si yo imparto clase, hago planeaciones igual que ellos, pero en mi caso me toca recibir la documentación de los alumnos para nuevo ingreso, digitalizarla, dar de alta al posible aspirante al sistema de administración que tenemos, revisar que su CURP [su DNI) esté correcta; después de eso tengo que encargarme de darles sus referencias en el banco, aplicar lo que es el examen de ingreso [...] esa es en la parte de dirección que hay que estar al pendiente de todo, ya que están los alumnos inscritos yo me encargo también del programa de becas [...] yo tengo que darlos de alta en el sistema y tengo que llenar actas en donde estoy solicitando becas para ellos, y si les autorizan esa beca yo me encargo de ir a las oficinas para recoger las tarjetas, entregárselas, recibir los acuses, digitalizarlos y subirlos también al sistema [EDRC2].

Esas actividades ciertamente no son todas, pero son muestra de que las variadas funciones que los docentes encargados realizan les llevan más tiempo de lo que al parecer le dedican a la docencia. Así, es dable pensar que esta queda desplazada por el cumplimiento de las cuestiones administrativas que, entre otras cosas, implica asegurar parte de la trayectoria del estudiantado, ya que las becas son indispensables para estos dado el contexto socioeconómico en el que viven. Además, por las múltiples actividades, la contratación de 30 horas no es suficiente ni para la docencia ni para lo referente a la cuestión administrativa, así que tienen que dedicar tiempo en el hogar para completar y terminar lo que no pueden hacer durante la jornada escolar, bien sea porque el tiempo no les alcanza o porque no hay las condiciones necesarias para cumplir su labor, como la conexión a internet e incluso señal telefónica que les comunique con las oficinas centrales de los TBC.

\section{Trabajo docente y flexibilización}

La forma en que los docentes relatan el trabajo que realizan al interior del aula refuerza la relación existente entre el perfil profesional y la flexibilidad de los procesos. El relato general de las prácticas docentes, aunque varían en tonalidades, no en el fondo o en el hilo conductor, que evidencia la falta de recursos de todo tipo, como bibliotecas o conectividad para hacerse de más información. Entonces el profesorado implícitamente acepta que las tareas o los trabajos de los estudiantes sean de poca exigencia, o bien contribuyen llevando alguna información que descargan de internet para que los jóvenes la trabajen. Sin embargo, lo anterior refleja un doble esfuerzo docente, por la falta de materiales y recursos y, también, de formación, pues no se perciben estrategias de estos para solventarlas bajo otro tipo de modalidades o estrategias pedagógicas.

Muchos de los procesos que se desarrollan en el TBC, aunque deberían de ser parte de la participación de diferentes instancias, son suplidos por el mismo profesorado, por ejemplo, tienen claridad de que reciben a los jóvenes con déficits de conocimientos que tendrían que haber adquirido en educación básica. La respuesta a lo anterior es, entonces, implementar diferentes estrategias para solventarlo, dedicando parte del primer semestre para regularizarlos; así, comentan: 
...tenemos la posibilidad, en el Telebachillerato nos dan el primer semestre para darles un curso propedéutico en donde los estamos estabilizando para que continúen [EDRC5].

Así, parte del semestre inicial lo dedican a intentar poner a los estudiantes al corriente de lo que deberían de saber al llegar al nivel medio, cosa que, en un corto tiempo, y con docentes no formados en dichas estrategias de recuperación o de aprendizajes bajo estas modalidades, es difícil que se logre.

Una vez que han superado esta etapa, y por lo comentado por los docentes, no es algo que lleva a que algunos queden fuera, sino a asegurar que tengan una trayectoria sin demasiados problemas. Lo anterior se une a otro tipo de procesos emparentados que muestran cierta flexibilidad del subsistema, ante las condiciones en las que arriban los estudiantes y por consideraciones del origen social de los jóvenes. El caso de la reprobación es un ejemplo claro de lo anterior, pues en los TBC no se encontraron problemas de este tipo; lo que argumentan los docentes es que ellos mismos los van regularizando cuando notan que andan mal, de tal forma que no tienen que llegar a lo que sucede en la mayoría de los subsistemas de educación media en México, en donde hay altos niveles de reprobación y esta se ha convertido en uno de los antecedentes principales para el abandono escolar (Weiss, 2015). Así, sostienen que ya no reprueban a los alumnos, sino que los tienen siempre trabajando y observando su desarrollo (EDRC4), y otro profesor comenta:

Se les da curso, de hecho, evitamos eso [la reprobación], vamos trabajando con ellos, en esas situaciones les vamos dejando trabajos extras, yo tutoreo y le digo "yo seré tu tutor", lo sentamos con el que sabe un poco más y vamos apoyándolos, esa es la forma en la que yo trabajo [EDATBC8].

Pero de trasfondo se encuentra un afán de flexibilizar los procesos, dado el contexto de las escuelas y las características de los jóvenes, por eso se refieren a la necesidad de contextualizar, a saber dónde están, y a mantener un nivel de exigencia de acuerdo con lo anterior.

\section{Conclusiones}

Los TBC como estrategia principal para el incremento de la cobertura en las zonas rurales parece haber abonado no solo a la respuesta de equidad para estas poblaciones que no habían tenido oportunidad de acceder a este nivel, sino para que, a la luz de los postulados de la nueva ruralidad, los jóvenes se incorporen a las dinámicas urbanas de empleo, contratación y estudios requeridos. Ciertamente hay unas nuevas reglas del juego (Giarracca, 2001), sin embargo, lo que parece que no ha cambiado es el proceso educativo trasladado de los modelos urbanos, imposibilitando un enfoque comunitario, trabajo colaborativo y estrategias propias de un currículo más flexible (RIER, 2018).

Si bien existieron referentes nacionales de política sobre los TBC, en los hechos parece que hubo una descentralización que desembocó en que cada entidad siguiera 
una lógica de desarrollo propia, cuya desembocadura ha sido, para el caso del profesorado, que hayan puesto el mayor de los esfuerzos y recursos para sacar adelante al subsistema.

Ante el reconocimiento de que no tienen la formación para desempeñarse como docentes, una de las estrategias que han seguido los mismos, y que refuerza la idea de que han sido estos los que han hecho posible en concreto su funcionamiento, es que han buscado formarse cursando incluso maestrías en Educación. En estos casos no hemos encontrado lo que otros estudios han mostrado al respecto (Leyva y Guerra, 2019), es decir, que ante un perfil alejado al tipo de actividad que desarrollan aparece el trabajo colaborativo y el apoyo entre pares.

La preeminencia de actividades administrativas es clara, se sobreponen a la función principal que es la docencia, es decir, se le da prioridad a la primera por la implicación que se puede tener, ya sea en el cumplimiento de los trámites como registro de asistencias y evaluaciones, hasta la posibilidad de las becas y el mantenimiento de la matrícula.

La formación inicial de los docentes parece estar condicionando fuertemente su práctica; el tipo de estilo docente (Yurén y Araújo, 2003) que asumen es el de centrarse en la especialidad de su formación inicial, ingenieros o administradores, y desde ahí emprender un cierto tipo de trabajo pedagógico con los estudiantes. Así, la flexibilidad con la que asumen su trabajo parece estar asociada a su perfil y formación inicial. Ante un contexto adverso donde los jóvenes no llegan con los conocimientos y habilidades requeridas para el nivel, intentan nivelar y al final facilitar los procesos educativos, desembocando en que no haya mejora en los aprendizajes del estudiantado, lo que configura un círculo pernicioso que, en el mejor de los casos, contribuye en poco a la formación de los jóvenes. Las mismas condiciones adversas que enfrentan los docentes terminan por impactar entonces en los aprendizajes y oportunidades de los estudiantes, sus condiciones de formación y de atención que reciben de la institución se reflejan en esta flexibilidad de los procesos educativos, en que prácticamente no exista la reprobación. Podemos caracterizar lo anterior como parte de todo un proceso endógeno que no está bajo la mirada externa, y que solo termina por hacerse evidente cuando se realizan algunas pruebas nacionales de evaluación de los aprendizajes, en las que los TBC consistentemente aparecen en los resultados más bajos (INEE, 2019).

La atención de los Telebachilleratos Comunitarios quedó en manos de responsables centrales en las entidades, con administraciones relativamente nuevas, con poca experiencia en educación media, y lo anterior se mostró en los procesos de contratación de los docentes, al delegar, en un inicio, esta labor en instancias externas, hasta ir generando capacidades propias. Pero también esto se ha visto en otros aspectos, como lo puede ser la actualización y capacitación de los docentes, pues no es casual que hayan sido estos los que han tenido que irse actualizando por su cuenta 
o estudiar posgrados relacionados con su función actual, pues han sentido que su formación inicial no respondía adecuadamente a las exigencias de ser profesores en estas instituciones. Esto, como dijimos, es parte de la conformación de una particular cultura docente que se ha ido generando entre el profesorado de los contextos rurales.

Los TBC se puede caracterizar como una opción marcada por la precariedad, no solo por la poca habilitación de sus docentes o la flexibilidad para los procesos formativos y los aprendizajes de los estudiantes, sino también por las condiciones de infraestructura, que no son solo materiales, sino que, como se ha mostrado, tienen implicaciones que, aunque simbólicas en el nivel de apropiación y de pertenencia e identidad, terminan por ser determinantes en los resultados de conjunto de los Telebachilleratos Comunitarios en la entidad.

Hay una clara desigualdad con la que llegan los estudiantes a este subsistema, y aunque los docentes hacen esfuerzos considerables, desde las condiciones institucionales y desde las posibilidades que les brinda su formación, no son suficientes para evitar que se siga dando la acumulación de desventajas de los jóvenes (Saraví, 2009). Así, estudiantado y profesorado comparten una trayectoria, los alumnos llegan con conocimientos deficientes para el nivel, y por eso los docentes expresan dedicarse durante el primer semestre a equiparar o ponerlos al corriente. Los profesores también llegan con un déficit de formación como docentes, y tienen que desarrollar un esfuerzo considerable para equipararse y ponerse al corriente con su profesión actual.

\section{REFERENCIAS}

Álvarez, J., y Jurgenson, G. (2003). Cómo hacer investigación cualitativa. Fundamentos y metodología. México: Paidos.

Angrosino, M. (2012). Etnografia y observación participante en investigación cualitativa. Madrid, España: Morata.

Backhoff, E., Guevara, G., Hernández, J., y Sánchez, A. (2018). El aprendizaje al término de la educación media superior en México. El Cotidiano. Revista de la realidad mexicana actual, (208), 7-20.

Bertely, M. (2000). Conociendo nuestras escuelas. Un acercamiento etnográfico a la cultura escolar. México: Paidós.

Bourdieu, P. (1987). Los tres estados del capital cultural. Sociológica. Revista del Departamento de Sociología, 2(5), 1-6.

Bourdieu, P. (1988). La distinción. Criterios y bases sociales del gusto. España: Taurus.

Cortés, S., Peniche, R., y Crescencio, C. (2019). Práctica de liderazgo escolar en un Telebachillerato Comunitario de alta eficacia. XV Congreso Nacional de Investigación Educativa. COMIE. Acapulco, Guerrero, México.

De Grammont, H. (2004). La nueva ruralidad en América Latina. Revista Mexicana de Sociología, (66), 279-300.

Espinoza, E. (2014). Dificultades en lengua escrita en español de estudiantes de Telebachillerato en contexto otomí [Tesis de maestría]. Universidad Veracruzana. Veracruz, México.

Estrada, M., y Alejo, S. (2018). Caracterización e impacto de los Telebachilleratos Comunitarios en Guanajuato. México: Colofón/Universidad de Guanajuato.

Estrada, M., y Alejo, S. (coords.) (2019). El Telebacbillerato Comunitario. De la cobertura a la búsqueda de equidad. México: Colofón/Universidad de Guanajuato.

Estrada, M., y Razo, F. (2019). Telebachilleratos comunitarios. La valoración de los jóvenes estudiantes. $\mathrm{R} a$ Ximbai, 15(3).

Giarracca, N. (2001). Prólogo. En N. Giarracca (comp.), ¿Una nueva ruralidad en América Latina? (pp. 11-14). Argentina: CLACSO/ASDI.

Goetz, J., y LeCompte, M. (1988). Etnografía y diseño cualitativo en investigación educativa. Madrid: Morata.

Guzmán, C. (2018). Avances y dificultades en la implementación del marco curricular común. Telebachillerato estatal, Educación media superior a distancia y Telebachillerato Comunitario. México: INEE. 
INEE [Instituto Nacional para la Evaluación de la Educación] (2019). Informe de resultados PLANEA EMS 2017. El aprendizaje de los alumnos de educación media superior en México. Lenguaje y comunicación y matemáticas. México: INEE.

Landín, X., y Arriaga, C. (2019). La secuencia didáctica como guia docente para brindar una educación de calidad en los Telebachilleratos Comunitarios. XV Congreso Nacional de Investigación Educativa. COMIE. Acapulco, Guerrero, México.

Leyva, Y., y Guerra, M. (2019). Las prácticas de docentes que trabajan en educación indígena, escuelas de organización multigrado, telesecundarias y Telebachilleratos Comunitarios en México. México: INEE.

Macías, A., y Valdés, M. (2014). Reconstrucción del rol docente de la educación media superior de enseñante tradicional a enseñante mediador. Sinéctica, (43).

Medrano, V. (2019). Los Telebachilleratos Comunitarios en México. En M. Estrada y S. Alejo (coords.), El Telebachillerato Comunitario. De la cobertura a la búsqueda de equidad (pp. 21-46). México: Universidad de Guanajuato/Colofón.

Pérez, E. (2004). El mundo rural latinoamericano y la nueva ruralidad. Nómadas, (20), 180-193.

Pérez, A. (2004a). La cultura escolar en la sociedad neoliberal. España: Morata.

Pérez, A. (2005). Las funciones sociales de la escuela: de la reproducción a la reconstrucción crítica del conocimiento y la experiencia. En G. Sacristán e I. Pérez, Comprendery transformar la enseñanza (pp. 17-33). España: Morata.

RIER [Red Temática de Investigación de Educación Rural] (2018, ago.). Propuestas para mejorar la atención educativa a poblaciones rurales en México (resumen del foro Propuestas para la educación rural para el sexenio 2018-2024. Universidad Iberoamericana, Ciudad de México). Recuperado de: https://www.uv.mx/ personal/romendoza/files/2018/12/PropuestasEducacion-Rural-final-1.pdf.
Rodríguez, S. (2018). La persistencia de la desigualdad social en el nivel medio superior de educación en México. Un estudio a nivel nacional. Perfiles Educativos, 40(161), 8-31.

Saccone, M. (2016). La educación media en tiempos de transformaciones: una mirada socio-antropológica hacia México y Argentina. Buenos Aires: CLACSO.

Salazar, P. (2007). Un estudio de estrategias y prácticas de los docentes en relación con las matemáticas de los profesores del Telebachillerato en el estado de Veracruz [Tesis de maestría]. Veracruz, México.

Saraví, G. (2009). Transiciones vulnerables: juventud, desigualdad y exclusión en México. México: Centro de Investigaciones y Estudios Superiores en Antropología Social.

SEMS [Subsecretaría de Educación Media Superior] (2016). Documento base. Telebachillerato comunitario. México: SEP.

SEMS (2018). Telebachilleratos comunitarios. México: SEP.

Strauss, A., y Corbin, J. (2002). Bases de la investigación cualitativa. Técnicas y procedimientos para desarrollar la teoría fundamentada. Colombia: Universidad de Antioquia.

Stufflebeam, D., y Shinkfield, A. (1987). Evaluación sistemática. Guia teórica y práctica. Barcelona: Paidós/MEC.

Weiss, E. (2015). El abandono escolar en la educación media superior: dimensiones, causas y políticas para abatirlo. En R. Ramírez (coord.), Los desafios de la educación media superior (pp. 81-159). México: Senado de la República/Instituto Belisario Domínguez.

Weiss, E. (2017). Estudio exploratorio del modelo de Telebachillerato Comunitario y su operación en los estados. México: INEE.

Weiss, E. (2017a). El Telebachillerato Comunitario. Una innovación curricular a discusión. Revista Latinoamericana de Estudios Educativos, 47(3-4), 7-26.

Yurén, M., y Araújo-Olivera, S. (2003). Estilos docentes, poderes y resistencias ante una reforma curricular. El caso de Formación cívica y ética en la escuela secundaria. Revista Mexicana de Investigación Educativa, 81(9), 631-652.

Cómo citar este artículo:

Estrada Ruiz, M. J., y Alejo López, S. J. (2021). Los docentes de Telebachilleratos Comunitarios, perfiles que orientan la práctica y flexibilización de los procesos formativos. IE Revista de Investigación Educativa de la REDIECH, 12, e1140. doi: 10.33010/ie_rie_rediech.v12i0.1140. 